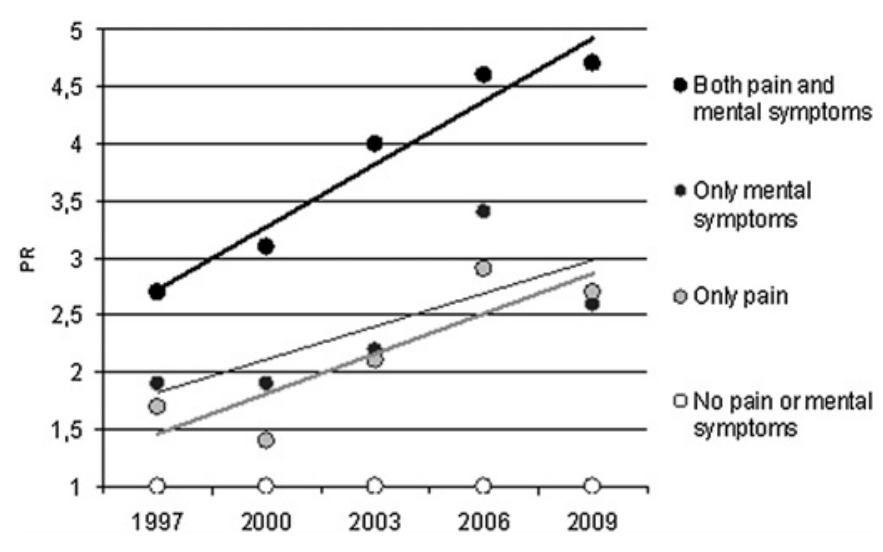

Abstract P2-452 Figure 1

Methods Five cross-sectional telephone surveys were conducted triennially in 1997-2009 among representative samples of working Finns ( $n=2000-2400 /$ survey year, total $N=11000$ ). The risk of reduced work ability related to musculoskeletal pain and mental symptoms was estimated with log-binomial regression for each survey year separately. Current work ability was assessed with a scale from 0 to 10 (reduced ability: 0-7). Musculoskeletal pain concerned prolonged or recurrent pain in the neck, shoulders/arms, wrist/fingers, low back, or hips/lower extremities. Mental symptoms included feelings of depression, tiredness, irritability, difficulties to concentrate, and sleep problems. Models were adjusted for age, gender, education, BMI, smoking, chronic illnesses, physical and psychosocial work exposures, accidents at work, threat of unemployment, and family problems.

Results One-month prevalence of co-occurring pain and mental symptoms as well as that of self-perceived work ability remained stable in 1997-2009. The adjusted prevalence ratios of reduced work ability related to having both pain and mental symptoms increased from 2.7 (95\% CI 1.7 to 4.2) in 1997 to 4.7 (95\% CI 2.9 to 7.8 ) in 2009 (Abstract P2-452 figure 1). Changes in the risk of reduced work ability associated with musculoskeletal or mental symptoms only were less marked.

Conclusions The considerable increase that occurred during 12 years in the risk of reduced work ability implies that workers with cooccurring musculoskeletal and mental symptoms presently experience less ability to work than before, irrespective of assessed changes in for example, physical or psychosocial work exposures.

\section{P2-453 NEONATAL SEPTICAEMIA IN INDIA: MAJOR CAUSE OF MORBIDITY \& MORTALITY AND CHALLENGE FOR HEALTH PROFESSIONALS}

doi:10.1136/jech.2011.142976l.81

G Mudey, ${ }^{*}$ N Tankhiwale, A Mudey. Jawaharlal Nehru Medical College, Sawangi (Meghe), Wardha, Maharashtra, India

Introduction Neonatal septicaemia is life-threatening emergency that demands urgent management and leading cause of neonatal mortality accounting nearly half of all neonatal deaths. Blood culture is gold standard method for diagnosis but changing pattern of organisms and frequent emergence of resistant bacteria causes difficulty in treatment. Non-specificity of symptoms creates difficulty in diagnosis of infections in the early stage. Present study was conducted with the objectives of isolation of bacteria from blood, their sensitivity and resistance pattern, correlation of maternal and fetal risk factors.

Methods Study was conducted in tertiary care centre on 210 cases of clinically diagnosed neonatal septicaemia admitted in NICU. Blood culture was done in all cases by conventional three subculture method and antibiotic sensitivity was done by Kirby Bauer disc diffusion. All cases were studied for maternal \& fetal risk factors.

Results Blood culture was positive in $49.05 \%$ cases. Klebsiella Pneumoniae was frequently isolated pathogen (63.11\%), followed by Escherichia coli (12.62\%) and Staphylococcus aureus (10.68\%). Gram negative isolates from enterobactericeae were $100 \%$ sensitive to imipenem followed by amikacin and cefotaxime. $S$ aureus isolates were $100 \%$ sensitive to vancomycin followed by amikacin and cloxacillin. The commonest maternal risk factors were muconium stained amniotic fluid $(42.72 \%)$, premature rupture of membrane (33\%), History of fever (20.39\%). Fetal risk factors commonly present were $79.61 \%$ low birth weight, $67.96 \%$ neonates were preterm and birth asphyxia (65.05\%).

Conclusion There is a need of continuous surveillance of the bacteriological profile and antimicrobial sensitivity pattern of neonatal septicaemia in each and every NICU.

\section{P2-454 ASSOCIATION BETWEEN HIV/AIDS RELATED ADULT DEATHS AND MIGRATION OF HOUSEHOLD MEMBERS IN RURAL RUFIJI DISTRICT, TANZANIA}

doi:10.1136/jech.2011.142976l.82

${ }^{1} \mathrm{~F}$ Murunga, ${ }^{*} \mathrm{H}$ Masanja. ${ }^{1}$ African Population and Health Research Center, Nairobi, Kenya; ${ }^{2}$ Ifakara Health Institute, Dar es Salaam, Tanzania

Introduction The dramatic increase in adult mortality attributable to HIV/AIDS in Sub Saharan Africa may increase the number of households that do not survive as a functional unit in the years to come. This report describes the extent at which these impacts are weathered by a rural community using data from the Rufiji HDSS in rural Tanzania.

Objectives The study describes adult mortality patterns in the area with an emphasis on the HIV/AIDS related adult deaths and the proportion of household members migrating following these deaths. Methods A total of 32787 households and 4603 adult deaths from 4019 households were recorded over the study period. Mortality trends and migration rates were computed while the association between adult mortality and out-migration of members assessed using Cox proportional Hazard model.

Results Adult deaths increase by about $9 \%$ the chance of a child to migrate within or without the DSA while HIV/AIDS adult deaths increase by a further 19 percentage point the risk of the child to migrate out of the DSA. Non-HIV/AIDS adult deaths also enhance the risk for female internal migration by $5 \%$ albeit hardly significantly (adj. HR 1.05; 95\% CI 1.0 to 1.10 , p value 0.05 ) but decreases the chance of male internal migration by $13 \%$ (adj. HR 0.87; $95 \% \mathrm{CI}$ 0.81 to 0.93 , p-value 0.01 ). HIV/AIDS adult death is strongly associated with out-migration of adults. The deaths female out-migration to $19 \%$ (adj. HR 1.19; $95 \%$ CI 1.09 to 1.30 , p value $<0.001$ ) and male migration to $30 \%$ increased risk (adj. HR 1.30; 95\% CI 1.16 to 1.45 , $\mathrm{p}$ value $<0.001)$

\section{P2-455 SLEEP DURATION AND RISK OF WEIGHT GAIN AND OBESITY: THE OHSAKI COHORT STUDY}

doi:10.1136/jech.2011.142976l.83

M Nagai, ${ }^{*}$ M Kakizaki, I Tsuji. Tohoku University Graduate School of Medicine, Sendai, Japan

Introduction Several studies from Western countries have reported that sleep duration is associated with weight gain or obesity. We examined the association between sleep duration and risk of weight gain or obesity in Japan.

Methods We combined data from two cohort studies. In 1994, we delivered a questionnaire to all individuals aged $40-79$ years who lived in the catchment area of Ohsaki Public Health Center. Of 